\title{
Indeterminism is a modal notion: branching spacetimes and Earman's pruning
}

\author{
Tomasz Placek • Nuel Belnap
}

Received: 9 June 2010 / Accepted: 25 October 2010 / Published online: 10 November 2010

(C) The Author(s) 2010. This article is published with open access at Springerlink.com

\begin{abstract}
The paper defends an Aristotelian notion of indeterminism, as rigorously formulated in the framework of branching space-times (BST) of Belnap (1992), against criticism by Earman's (2008) based on a model-theoretic characterization of indeterminism. It delineates BST branching against the background provided by Earman's (2008) distinction between individual versus ensemble branching. Partly in order to motivate our responses to Earman, it describes a construction of physically motivated BST models, in which histories are isomorphic to Minkowski spacetime. Finally it responds to Earman's criticisms leveled against BST by addressing a topological issue, the question of an actual future, the past/future asymmetry, and some semantical questions.
\end{abstract}

Keywords Indeterminism/determinism - Spacetimes · Branching space-times · Tenses

In current debates two different concepts of (in)determinism have been used, each having a different historical origin and each being applied in different fields. On the one hand, there is an everyday natural notion of (in)determinism, discussed first by Aristotle, and illustrated by alternative possible futures, one with a sea battle and the other without. On the other hand, there is a tradition started by Laplace, of characterizing

T. Placek (凶)

Jagiellonian University, Kraków, Poland

e-mail: Tomasz.Placek@uj.edu.pl

N. Belnap

Department of Philosophy, University of Pittsburgh, Pittsburgh, PA, USA

e-mail: belnap@pitt.edu 
(in)determinism in terms of laws and predictions. This latter concept was rigorously analyzed by the logician, R. Montague, in terms of models of a theory that are alike up to a certain time, and then diverge. By replacing "models of a theory" by "possible worlds", Lewis later adapted Montague's analysis to general philosophy; in turn, philosophers of physics, especially Earman, have used Lewis-inspired concepts of (in)determinism.

"Branching space-times" is a rigorous attempt to analyze the natural (Aristotelian) notion of indeterminism. The theory appeared in Belnap (1992) [see also Belnap 2003a] (henceforth BST92 for the publication, and BST for the theory), and has since been developed by several philosophers in a variety of directions. For relevant publications, see items in the list of references by Belnap et al., by Müller et al., and by Placek et al. BST92 built on earlier work on the "branching times" (henceforth BT) representation of indeterminism that goes back to a passing suggestion in an unpublished letter from Kripke to Prior dated September 3, 1958, ${ }^{1}$ to the short discussion in Prior (1967), and to its working out in Thomason (1970). For our own earlier work on BT, we refer to Facing the Future (Belnap et al. 2001, henceforth, FF).

Recently, BST has been attacked by Earman, a leading figure in the philosophy of physics. Earman (2008) (henceforth Pruning), finds BST troublesome in several ways, some minor, others major. The major objections are as follows. (1) It is not clear what kind of branching BST assumes, where one kind, individual branching, is in conflict with general relativity. (2) On the BST account, spacetimes seem to violate the Hausdorff property. (3) BST opposes the doctrine that "(as of now) there is [a] fact of the matter as to which of the possible futures is the actual future" (p. 190). (4) In contrast to time-reversal symmetric physics, BST assumes a past versus future asymmetry. (5) In addition to these complaints, Earman advances a certain semantical rule that is at variance with BST semantics.

It seems to us that these objections and perhaps misunderstandings stem from differing notions of (in)determinism, which in turn underlie differing versions of branching. So we begin our essay in response to (1) in (Sect. 1) with an attempt to clarify Earman's diagnosis of a kind of branching assumed in BST. Then, to prepare for answering the remainder of Earman's objections, in Sect. 2 we offer an overview of BST. To emphasize how naturally a BST model arises out of field-theory inspired considerations, in Sect. 3 we develop an account of particular BST models, known as Minkowskian Branching Structures (MBS's). In Sect. 4 we reply to Earman's criticisms (2)-(5). Finally, our views on indeterminism, and why we think Earman is seriously mistaken, are in Sect. 5.

\section{Three types of branching}

Pruning begins its discussion of branching by defining three types of branching, which Earman labels "ensemble branching," "individual branching," and "Belnap branching." To explain, branching is a means of representing incompatible possible

$\overline{1 \text { This is mentioned by Øhrstrøm }}$ and Hasle (1995, p. 173). 
courses of events, and should be contrasted with forks used to represent spatiotemporal relations.

\subsection{Ensemble and individual branching defined}

The first two types are defined with reasonable precision:

"Ensemble branching" is defined by four features.

1. We have a "collection of spacetime models (worlds, histories, ...)" (p. 188). The collection is the "ensemble"; note especially that its members are spacetime models in a standard sense; that is, each is a structure that can serve as a model in Tarski's sense of a spacetime theory in Einstein's sense. ${ }^{2}$

2. The collection contains spacetime models that are isomorphic over some region (typically for all times before a given time), but non-isomorphic elsewhere.

3. The "isomorphism at issue may be construed either as literal identity or as a counterpart relation" (p. 188) in something like the sense of Lewis (1986).

4. There is no "branching structure in any individual spacetime model" (p. 187).

For most spacetime theories, their models (aka spacetime models) can be viewed as structures of differential geometry. A spacetime model so understood is a differential manifold $\mathcal{M}$ with a collection of object-fields defined on $\mathcal{M}$; these object-fields characterize the structure of spacetime or the matter-fields (cf. Pruning, footnote 3).

"Individual branching," in contrast, "involves branching structures in individual spacetime models" (p. 187). This is the only distinguishing feature that decides between individual branching and ensemble branching. In particular, in Earman's characterization, the choice between the identity relation and other kinds of isomorphism for an adequate relation between spacetime models, is not relevant to the distinction. This may come as a surprise to readers of David Lewis, who objected to the literal overlap of initial segments of worlds, while recommending a similarity of initial segments as an adequate concept to characterize (in)determinism. ${ }^{3}$ For Lewis the issue "what kind of isomorphism" was significant for the characterization of indeterminism. We may, however, put that aside; the chief point is that in Pruning, Earman distinguishes three types of branching that might be invoked in considering indeterminism. We recapitulate the first two.

Ensemble-branching is ensemble branching in the sense given by Earman's clauses (1)-(4) above. The central idea is that indeterminism is represented by branching that is between rather than within individual spacetime models.

\footnotetext{
2 We attribute this sense of "model" to Earman in virtue of Earman (1986, pp. 20-21), which refers to the logician, Montague (1962). Earman refers there to Montague's model-theoretic analysis of (in)determinism of theories (understood as syntactic objects), takes possible worlds to be counterparts of models, and then explicates (in)determinism in terms of possible worlds.

3 Lewis (1986) called the recommended option "divergence," based on similarity of initial segments, in contrast with "branching," based on overlapping initial segments.
} 
Individual branching is individual branching in Earman's sense. The idea here is that an attempt is made to represent indeterminism by branching within an individual spacetime model.

We will also classify theories as "ensemble-branching" or "individual-branching," according to the type of branching involved. Earman argues that (a) ensemble branching gives a good, scientifically respectable account of indeterminism, whereas (b) any individual-branching theory is bound to be unpalatable in some important fashion. The argument for (a) lies in other work by Earman, principally in his prizewinning book, Earman (1986), but see also the more recent Earman (2007). Much of Pruning is devoted to (b), which is largely outside the scope of this essay.

\subsection{BST branching}

Earman defines his third type of branching, "Belnap branching," only by vague reference to "the Belnap school of branching spacetimes" (p. 187). In order to avoid unfortunate personalization, we will call this third type "BST branching." We will keep the definition historical: BST branching is the kind of branching of overlapping "histories" that is axiomatized with mathematical precision in BST92 and subsequent papers. See Sect. 2.3 for a notation-free but exact list of axioms.

Our major discussion of BST branching occurs in Sects. 2 and 3. Here we insert a few paragraphs on the topic, so as to put the extended discussion into a proper context.

In Pruning Earman complains at length about BST. ${ }^{4}$ In some passages, Earman tends to classify BST as postulating the objectionable individual branching, which he intends as a serious complaint. Sometimes, however, he merely complains that he is "unable to get a fix on" BST branching (p. 192), which Earman characterizes as lying "somewhere between" ensemble and individual branching, at a location that "is not easy to pin down" (p. 189). Since BST92 offers a carefully crafted rigorous theory living up to the standards of mathematical logic, it is difficult for us to appreciate Earman's hesitation. In the context of what it calls a Minkowski branching spacetime (Definition 16 of Belnap (1992)), BST92 treats as interchangeable the words "history," rigorously defined as a possible course of events, and "spacetime," left undefined in terms of the primitives of BST92, but evidently intended to connote a spacetime in the sense of special relativity. BST does not tolerate any branching within an individual history. Rather, as in ensemble-branching theories, all branching is between histories_-which, as noted, are imagined by BST as individual spacetimes. In this respect, then, BST is rather like an ensemble-branching theory.

What might give rise to naive wondering if BST is, after all, a individual-branching theory is this: Each individual model of the theory of BST involves branching. BST is not, however, of the individual-branching type. The reason is this: An individual

\footnotetext{
4 We confess to a certain ambivalence about being one of the targets of Pruning: On the one hand, we are honored by having a truly distinguished philosopher of science focus his attention on BST; on the other hand, we are disappointed that, judging by Pruning, Earman seems to have looked at only some thoroughly informal paragraphs of our papers, giving not the slightest hint that he has considered any part of the extensive technical development that constitutes the heart of our work.
} 
model of BST is neither a single history, nor an individual spacetime. In short, a model of BST in the proper logical sense is not, nor is it intended to be, a spacetime model in Earman's sense. An individual model of BST involves many histories (read "space-times"). Here we are using "model" in the same standard logical sense as does Earman, who derives his usage from Montague. The difference between an individual spacetime model and a model of BST is that the former has power to represent neither alternative possibilities nor other modal notions. Put it this way: A single, individual model of BST theory represents many pairwise-incompatible branching courses of events (each course of events imagined as a spacetime with content), whereas an individual model in Earman's sense represents a single course of events. ${ }^{5}$ Thus, BST has to be counted as neither an ensemble-branching nor an individual-branching theory.

To repeat, in BST indeterminism is represented by branching in an individual structure, as in individual branching, but the model is quite unlike a spacetime model. It is in fact a more complex structure (a "world") that involves many pairwise-branching "histories," each history construed as a spacetime, as in ensemble branching. A key feature is that a BST-branching physical theory has language that can describe branching without ascending to a metalanguage. ${ }^{6}$ We defer discussion of BST branching to Sect. 2.

\section{BT/BST branching}

"Branching time" (BT) is an axiomatic theory postulating that the world is a tree of "moments," the latter being envisaged as instantaneous "superevents" that, although instantaneous, are spread out over all of space. ${ }^{7}$ BST theory is a refinement of BT theory.

\subsection{BST: Our World and its point events}

The construction of BST92 begins with a set, called Our World, of possible point events - instead of BT-moments - each of which is an ideally small event that is in a "suitable external relation" (Lewis) to us at present. Speaking of his own worlds, Lewis writes, “... each world is interrelated (and is maximal with respect to such interrelation) by a system of relations which, if they are not the spatiotemporal relations rightly so called, are at any rate analogous to them" (Lewis 1986, p. 75). We hasten to accept this account of a "world," adapting it to BST by taking the relation in question to be the reflexive, symmetric, and transitive closure of the fundamental causal-temporal ordering, $\leqslant$, of possible point events. To accommodate indeterminism, the set includes point events that either belong to the future of possibilities of

\footnotetext{
${ }^{5}$ Cf. the distinction between "modally thick" and "modally thin" structures made in Sect. 2.5. Observe in particular that BST publications cannot be understood if one follows Earman in casually identifying "worlds" and "histories" (p. 188).

6 We count talk about ensembles of models as "metalinguistic," since, although they can be defined as collections of nonlinguistic set-theoretical structures, they make little sense except in their relation to theories.

7 See FF for history and references.
} 
here-now, or belong to the future of possibilities of some point event in the (causal) past of here-now. Of some events from that latter category, we may say, from our present standpoint, that they could have occurred (it was possible that they would occur). We may thus visualize a point event as linked to us at present by zigzagging lines, each line representing a causal-temporal order, in part analogous to the order familiar from special relativity. Note that we use here an indexical language, since our notion of possibility is fundamentally indexical. After this explanation, we choose to avoid explicitly indexical language. Thus, we will for brevity say "point event" rather than "possible point event." With this understanding, we say that Our World, abbreviated in technical passages by $O W$, is the totality of point events, partially ordered by a causal-temporal ordering $\leqslant$, with the ordering $e \leqslant e^{\prime}$ understood as " $e$ ' is in a possible future of $e$ " and, equivalently, " $e$ is in the (settled) causal past of $e^{\prime}$." The locution "Our World" is intended to emphasize that as far as BST goes, only one "world" is postulated. That one world, Our World, suffices for possibilities based in reality. We of course have no reason to block investigation of the idea of a "plurality of worlds" in connection with unreal possibilities or merely imagined possibilities. It is just that, rightly or wrongly, BST has nothing to say about them. ${ }^{8}$

There is a pre-geometrical flavor to the concept of point event, to be seen in examples such as an elementary particle impinging upon an ideally smooth surface. The elementary status of the particle indicates that this event is both very, very tiny, and not further analyzable. The event of the elementary particle impinging upon a smooth surface has no parts (Euclid); it is thus a point event. It should be clear that point events are not to be identified with their locations.

\subsection{BST: histories}

There is on this account, and in contrast to Lewis, only one world. To capture indeterminism, $O W$ needs to feature at least two "histories" (aka really possible courses of events), even though there is no postulate to this effect. In BST, the notion of "history" is defined in terms of the two primitives, $O W$ and $\leqslant$. In analogy to many other possible-worlds-like theories, there are two ingredients to the notion of "history": consistency and maximality. For a criterion of consistency of two events, BST takes the existence of a (joint) upper bound of these two events. This decision is motivated by the natural idea that the upper bound of two events serves as a perspective from which the two events could have been seen to have occurred jointly. Since a subset $A$ of $O W$ is called directed (sometimes "upward directed") if any two of its elements have an upper bound in A, a history is defined as a maximal upward directed subset of $O W$. Thus, significantly, BST histories are certain sets of point events. In BST92, every two histories overlap, which tallies nicely with the notion that every point event in $O W$ is in a "suitable external relation" to us at present: Overlap evidently guarantees that any two point events can be linked by a zigzagging causal line in the shape of an "M".

\footnotetext{
8 The purposes of Pruning permit using "model," "world," and "history" interchangeably (p. 188), and we may add "spacetime model." That won't do for understanding BST.
} 


\subsection{BST: axioms}

BST92 is mathematically rigorous: It presents an axiomatic theory, essentially second order because of the idea of histories. It is not our business here to explain BST axioms, few though they are, except to the extent required for clearing up the confusions and answering the complaints of Pruning. It is important, however, that BST is a mathematically exact theory, and to make this evident, we briefly state its axioms: $O W$ is a nonempty set, $\leqslant$ is a dense partial order on $O W$, each lower bounded chain has an infimum, and if upper bounded, has a supremum in each history containing it, $O W$ has no maximal elements, and finally, for histories $h_{1}, h_{2}$, if $E$ is a nonempty lower bounded chain in $h_{1} / h_{2}$, then some lower bound of $E$ is maximal in $h_{1} \cap h_{2}$. This last axiom (called the prior choice principle) entails that every two histories overlap and, moreover, that there is at least one element maximal in their overlap. Such an element we call a choice event of these histories.

\subsection{BST: space-like relatedness}

BST has natural concepts of (in)consistency and of space-like relatedness: Two events are consistent iff they jointly belong to some one history; otherwise they are inconsistent; and two events are space-like related iff they are consistent but incomparable with respect to the ordering $\leqslant$ on $O W$.

\subsection{BST: modal thickness and thinness}

Note the radical difference between the concept of $O W$ and the concept of a history. $O W$ contains every event that was, is, or will be really possible (this being said from our present standpoint). ${ }^{9}$ Accordingly, in general $O W$ has many (alternative) possible histories.

Having this modal aspect in mind, we say that $O W$ is modally thick. In contrast, a history represents (ideally, in minute detail) some particular scenario. Within a fixed history, there are no alternative possibilities, since possibilities alternative to those occurring in a given history must occur in other, alternative histories. We thus say that a single history is modally thin.

\subsection{BST: applications}

BST92 has been developed with an eye to two sorts of application. It has been applied to rigorously analyze (in)determinism and some consequences thereof, understood as features of the world rather than features of language, theories, laws, or our knowledge of the world. Here belong BST theories of causation and of agency in an indeterministic

\footnotetext{
9 In contrast to Lewis, we do not deal with fanciful notions of possibility. Xu (1997) coined the term, "possibilities based in reality"; that is what BST means to treat. This perhaps comes through most clearly in the BST theory of causae causantes, or "originating causes" as developed in Belnap (2005b).
} 
world, as well as the theory of causal probability spaces. ${ }^{10}$ These theories are intended as objective in the same sense that a theory of physics may be intended as objective. The second application is to provide formal semantics for a language with - in addition to standard truth-functional and quantificational operators-temporal or spatiotemporal operators, modalities whose semantics involves quantification over histories, and indexicals. ${ }^{11}$ It is striking that although these are large tasks, BST is frugal both with respect to primitive concepts and with respect to postulates. Nevertheless, BST lacks many features. Importantly, it has (for better or worse) no concept of laws of nature, although it is laws-friendly, since it has modalities and propositions, both rigorously defined-see e.g. Belnap (2002b). Its extension by agentive concepts is natural and straightforward, but the attempt to add mentalist concepts rapidly takes one away from its fundamental commitments.

\subsection{Spatiotemporal locations}

Here we will say a word about the relation between BST histories and spatiotemporal locations. The entire next section is devoted to a particular class of BST models, in which histories are like Minkowski spacetime.

To clarify a relation between histories and spatiotemporal locations, recall that BST histories are particular sets of point events. In everyday discourse, we ask what would have occurred at a given time, if things had gone differently at some junction in the past. Similarly, we wonder what would have happened in a given spatial location, if things had gone differently. We thus have a notion of events, belonging to various histories, yet occurring at the same instant, or in the same spatial location, as a given event. Having learned the relativity lesson, we should not separate time and spatial location, aiming instead to capture the concept of "spatiotemporal locations," st-locations, for short. Also, the "what would ..." questions mentioned above make sense in limited circumstances only: if we consider some extravagant evolution of the universe, say, a big crunch five seconds after the big bang, it is doubtful that we can profitably wonder what would have occurred at our present st-location, if that other evolution had taken place. The alternative does not seem to make sober sense. Thus, the histories considered should be in some sense similar to allow for common st-locations. Accordingly, only particular BST models permit the introduction of a set of common st-locations.

In this vein, Müller (2005) defines a set $S$ of common st-locations in a BST model $\langle W, \leqslant\rangle$ as a set of equivalence classes on $O W$ that satisfy certain conservativeness conditions with respect to the ordering $\leqslant$, namely (using $\exists$ ! for existence and uniqueness):

$$
\forall s \in S \forall h \in \text { Hist } \exists ! x \in W: x \in s \cap h \text { and }
$$

\footnotetext{
10 Cf. Belnap (2005a,b), Placek (2004), Weiner and Belnap (2006), and Müller (2005).

11 Cf. Müller (2002), Belnap and Green (1994), Belnap (2007), and Placek and Müller (2007).
} 


$$
\begin{aligned}
& \forall s_{1}, s_{2} \in S \forall h_{1}, h_{2} \in \text { Hist } \forall x_{1}, x_{2}, y_{1}, y_{2} \in W: \\
& \left(\left(x_{1} \in s_{1} \cap h_{1} \wedge x_{2} \in s_{2} \cap h_{1} \wedge x_{1} \leqslant x_{2} \wedge y_{1} \in s_{1} \cap h_{2} \wedge y_{2} \in s_{2} \cap h_{2}\right)\right. \\
& \left.\quad \rightarrow y_{1} \leqslant y_{2}\right) .
\end{aligned}
$$

As Müller shows, there are BST models that do not allow for the introduction of common st-locations. In his illustration, a base set of a BST model consists of pairs of real numbers and has two histories which overlap below and in the wings of point $\langle 0,0\rangle$, yet dramatically diverge above this point, as one is isomorphic to the positive real half-line and the other to a region of $\mathbb{R}^{2}$.

\section{Physically motivated BST models}

Until now there has been no link between abstract set-theoretical constructions of BST and models of mathematical physics. This predicament has led to complaints as to where in physical models there are objects postulated by BST, for instance choice points (compare a quote from Earman, as discussed in Sect. 5). To repair this unfortunate situation, in this section we construct physically motivated BST models, in which histories are isomorphic to Minkowski spacetime. The main news is how naturally a BST model arises from field-theory inspired considerations.

Having said so, we rush to emphasize that we are not so foolish as to believe that every spacetime of physics can be re-described in a BST model. As stated in BST92, some general relativistic spacetimes appear to conflict with BST definitions or axioms, and at present we do not know how to modify these to make them cohere with models of general relativity. In particular, the strict partial ordering $\leqslant$ of BST conflicts with causal loops of some models of general relativity. Also, as explicitly stated in BST92, the idea of taking a downward fork as a criterion for the involved events to be in one history, seems to be in conflict with spacetimes with black holes. ${ }^{12}$ We will show in the next section that there are nevertheless BST models with histories isomorphic to Minkowski spacetime, which suggests that BST models for general relativistic spacetimes with weak gravitational fields can also be constructed.

\subsection{Minkowskian branching structures}

We turn now to constructing a special class of BST models, called MBS's, which are more relevant (we believe) to physics. ${ }^{13}$ First, each history of an MBS is iso-

\footnotetext{
12 B. Roberts remarked to us that the mentioned conflicts seem always to involve global features of spacetime topology. As for local features of such troublesome spacetimes, they are reasonable in the sense that there exists an open neighborhood around every point that can be embedded in $\mathbb{R}^{4}$. Thus, BST can be interpreted minimally as an attempt to describe the local indeterminism in our world in our vicinity. Another option is to refurbish BST by replacing a global notion of history by some local notions; a first attempt in this direction is a theory of possible branching continuations, cf. Placek (2010).

13 The notion of a Minkowskian Branching Structure was first introduced informally in Belnap (1992) as a BST model in which every history is a Minkowski spacetime. Placek (2000) first attempted to produce a BST model out of (copies of) Minkowski spacetime, but failed. The first correct construction of MBS's (but with finitistic assumptions) is in Müller (2002).
} 
morphic to the Minkowski spacetime. Secondly, the construction proceeds in terms of assignments of physical properties (typically, strengths of physical fields) to spacetime points, which is more palatable to physicists' taste than the abstract axioms of BST theory. Furthermore, MBS's permit the introduction of common st-locations. On the other hand, we shall see that for the construction to succeed, some additional physical conditions must be satisfied.

Recall that Minkowski spacetime is a four dimensional real vector space, with the distance function $D_{M}^{2}: \mathbb{R}^{4} \times \mathbb{R}^{4} \rightarrow \mathbb{R}$ defined as follows (for $x, y \in \mathbb{R}^{4}$ ):

$$
D_{M}^{2}(x, y):=-\left(x^{0}-y^{0}\right)^{2}+\sum_{i=1}^{3}\left(x^{i}-y^{i}\right)^{2} .
$$

The Minkowskian ordering, $\leqslant_{M}$, is defined in the usual way as follows:

$$
x \leqslant M \quad y \text { iff } D_{M}^{2}(x, y) \leqslant 0 \text { and } x^{0} \leqslant y^{0} .
$$

Two points $x, y \in \mathbb{R}^{4}$ are space-like related in the Minkowski spacetime ('SLR $M$ ' for short) iff neither $x \leqslant_{M} y$ nor $y \leqslant_{M} x$. Naturally, $x<_{M} y$ iff $x \neq y$ and $x \leqslant_{M} y$.

Many physical theories ascribe physical properties, typically strengths of physical fields, to points of a spacetime, or can be viewed as involving such an ascription. If the underlying spacetime is Minkowski spacetime, the properties are ascribed to points of Minkowski spacetime, i.e., elements of $\mathbb{R}^{4}$. A minimal (necessary) condition for a theory to exhibit indeterminism is that it allows for many "scenarios" ascribing alternative possible properties to points of Minkowski spacetime. In other words, one point of $\mathbb{R}^{4}$ may have alternative properties assigned, depending on the scenario.

Ultimately we will define an MBS as a triple $\mathfrak{M}=\langle\Sigma, F, P\rangle$ (cf. Definition 8). We will begin by partly characterizing $\Sigma, F$, and $P$. To help capture abstractly the informal concept of possible "scenarios," we assume a non-empty set $\Sigma$ of "scenarios." We let $\sigma, \eta, \gamma$ range over $\Sigma$. We want to think of a scenario as Minkowski spacetime filled with some "content," where the content of a scenario should be representable by an attribution of properties to each Minkowski spacetime point. That is, the content of a single scenario, $\sigma$, may be represented by a function in the set $\mathbb{R}^{4} \rightarrow \mathcal{P}(P)$, where $P$ is a nonempty set of properties attributable to points of $\mathbb{R}^{4}$. Our purposes do not require putting any structure on $P$. A system of such contents can then be represented by a global attribution of properties $F: \Sigma \times \mathbb{R}^{4} \rightarrow \mathcal{P}(P)$. We will call such an $F$ a "property attribution on $\Sigma$ and $P$," noting that it is in effect a modal notion since referring to alternative possible properties for the same spacetime point. Writing $\sigma x$ for a pair from $\Sigma \times \mathbb{R}^{4}$, we may read " $F(\sigma x)$ " as "the set of properties instantiated at spacetime point $x$ in scenario $\sigma$." We informally think of the set in question as containing compatible properties only.

This function evidently dictates for each spacetime point, $x$, whether two scenarios, $\sigma, \eta$, are qualitatively the same there $(F(\sigma x)=F(\eta x))$ or not $(F(\sigma x) \neq F(\eta x))$. Clearly, many property attributions yield a pattern of scenarios without any similarity to what one might call indeterminism. Indeed, there is a consensus that indeterminism 
involves many scenarios that agree over some region (typically, an initial region) and then disagree over some (typically, later) region. In what follows, we will single out some special property attributions (which we will call proper property attributions), which seem reasonable, intuitively speaking. We will find that we are led to a pattern of indeterminism that is describable by branching space-times. This means that we will derive from an MBS $\mathfrak{M}=\langle\Sigma, F, P\rangle$ a BST-like Our World $\left\langle B, \leqslant_{R}\right\rangle$, and BST-like notions of history and choice event, and show that the BST axioms are satisfied in the defined model.

Let us now turn to a task of defining a proper property attribution, $F$. A part of this task is to single out a set of particular points of $\mathbb{R}^{4}$, to be interpreted, in loose talk, as locations of chanciness, as where the scenarios diverge. We will call such points "splitting points."

We first assume that every two scenarios differ somewhere, i.e.,

$$
\forall \sigma, \eta \in \Sigma\left(\sigma \neq \eta \rightarrow \exists x \in \mathbb{R}^{4} F(\sigma x) \neq F(\eta x)\right)
$$

where $F$ is a property attribution on $\Sigma$ and $P$.

This assures us that every two scenarios are qualitatively different; we further require that the pattern of differences for two scenarios be rather special: We postulate that for every two scenarios there is (at least one) point $s \in \mathbb{R}^{4}$ such that the scenarios agree in the past of $s$ and at $s$, but disagree somewhere in the immediate future of $s$. A point satisfying these two conditions will be defined as a splitting point for the two scenarios. ${ }^{14}$

The "past and present agreement" (PastPA) clause, that is, "past and present agreement of scenarios $\sigma, \eta$ from a spacetime point $s$," is easy to state: Where $F$ is a property attribution on $\Sigma$ and $P$, and $\sigma, \eta \in \Sigma$, and $s \in \mathbb{R}^{4}$

$$
\forall y \in \mathbb{R}^{4}(y \leqslant M s \rightarrow F(\sigma y)=F(\eta y)) .
$$

The "immediate future disagreement" (ImFDis) clause, that is, "disagreement of $\sigma, \eta$ immediately future to s," needs, however, to be handled with more delicacy. The nub is that to say that $\sigma, \eta$ split at $s$ is by no means to say that their contents differ at every spacetime point above $s$. Far from it: It makes perfect sense, for example, that they reconvene qualitatively at some point $x$ above $s$, and even remain largely the same in content for ever after (see Footnote 13). It would not, however, look reasonable if over some spatiotemporal stretch immediately above a splitting point for two scenarios there were no qualitative differences between these scenarios. Just as a point of $\mathbb{R}^{4}$ at which $\sigma, \eta$ are different in content is not a splitting point, so also a point that is not immediately followed by qualitative differences between two scenarios is not, after all, the point at which they split. In other words, for $s$ to be a splitting point between two scenarios $\sigma, \eta$ requires not only (PastPA), but also, no matter how close a future point

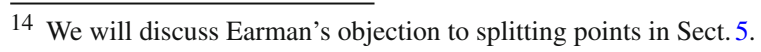


$x$ is to $s$, that there is always an even closer future point at which the two scenarios disagree in content (immediate future disagreement) ${ }^{15}$ :

$$
\forall x \in \mathbb{R}^{4}\left(s<_{M} x \rightarrow \exists y \in \mathbb{R}^{4}\left(s<_{M} y<_{M} x \wedge F(\sigma y) \neq F(\eta y)\right)\right) . \quad \text { (ImFDis) }
$$

The following definitions are therefore well-motivated.

Definition 1 (Splitting points) Given $\mathfrak{M}=\langle\Sigma, F, P\rangle$, where $F$ is a property attribution on $\Sigma$ and $P$ and $\sigma, \eta \in \Sigma$ and $s \in \mathbb{R}^{4}, s$ is a splitting point between scenarios $\sigma, \eta$ iff $s$ satisfies the condition (PastPA) of past and present agreement and the condition (ImFDis) of immediate future disagreement.

$S_{\sigma \eta} \subseteq \mathbb{R}^{4}$ is defined as the set of all splitting points between scenarios $\sigma, \eta \in \Sigma$.

Splitting is a qualitative notion that is derived from differences of properties in scenarios, in contrast to the cause-like notion of choice points of BST92.

Definition 2 (Proper property attribution) Given $\mathfrak{M}=\langle\Sigma, F, P\rangle, F$ is a proper property attribution on a set of scenarios $\Sigma$ and a set of properties $P$ iff $F: \Sigma \times \mathbb{R}^{4} \rightarrow$ $\mathcal{P}(P)$, and $F$ satisfies the condition SDiff that scenarios differ, and for all $\sigma, \eta \in \Sigma$,

(1) $\forall x \in \mathbb{R}^{4}\left(F(\sigma x) \neq F(\eta x) \rightarrow \exists s \in \mathbb{R}^{4}\left(s<_{M} x \wedge s \in S_{\sigma \eta}\right)\right)$, and

(2) for every lower bounded chain $E$ in $\left\langle\mathbb{R}^{4}, \leqslant_{M}\right\rangle$, if $\forall x \in E \exists s\left(s \in S_{\sigma \eta} \wedge s<_{M} x\right)$, then $\exists s_{0}\left(s_{0} \in S_{\sigma \eta} \wedge \forall x\left(x \in E \rightarrow s_{0}<_{M} x\right)\right)$.

Observe that the first clause links points of qualitative difference to splitting points: below each point of difference there is a splitting point. The second clause is a constraint on a set of splitting points: if there is a splitting point below each element of a lower bounded chain, then there is a splitting point below the whole chain.

We show now that for any $\sigma, \eta \in \Sigma$, the set $S_{\sigma \eta}$ of splitting points induced by a proper property attribution on $\Sigma$ and $P$ has some natural properties. Two of these properties are stated in terms of what we soon prove to be a region of $\mathbb{R}^{4}$ where the two scenarios are qualitatively the same; but first the definition. ${ }^{16}$

Definition 3 (Region of overlap) Given $\mathfrak{M}=\langle\Sigma, F, P\rangle$, for $\sigma, \eta \in \Sigma, R_{\sigma \eta}:=$ $\left\{x \in \mathbb{R}^{4} \mid \neg \exists s\left(s<_{M} x \wedge s \in S_{\sigma \eta}\right)\right\}$

We will show as part of our proof of Fact 4 just below that $R_{\sigma \eta}$ is indeed a region of $\mathbb{R}^{4}$ where the two scenarios are qualitatively the same.

Fact 4 Assume that $F$ is a proper property attribution on $\Sigma$ and P. Then:

\footnotetext{
15 We are quick to point out that ImFDis has a consequence that there must be infinitely many points of disagreement located on the future light-cone of a splitting point $s$ in each direction. As one of our referees indicated, one can tell a cogent, or apparently cogent, physical story about a photon taking one of two alternative paths from a splitting point $s$, which hardly calls for filling up the future light cone of $s$ with points of disagreement in every direction, as ImFDis requires. Nevertheless, our proofs rely on ImFDis; we leave it for future research to construct MBS's without this consequence.

16 Note that we write "a region," not "the region"; there is no implication that the two scenarios are qualitatively different everywhere else. Remember that we allow for split scenarios to largely reconvene. Regions of overlap were first introduced by Müller (2002).
} 
(i) $\sigma \neq \eta \rightarrow S_{\sigma \eta} \neq \emptyset$;

(ii) $S_{\sigma \eta}=S_{\eta \sigma}$;

(iii) $\forall s, s^{\prime} \in S_{\sigma \eta}\left(s \neq s^{\prime} \rightarrow s S L R_{M} s^{\prime}\right)$;

(iv) $x \in R_{\sigma \eta} \rightarrow F(\sigma x)=F(\eta x)$, and

(v) $\forall \sigma, \eta, \gamma \in \Sigma R_{\sigma \eta} \cap R_{\eta \gamma} \subseteq R_{\sigma \gamma}$.

Proof Ad (i): Immediate from Definition 2.

Ad (ii): Immediate from Definition 1.

Ad (iii): Assuming $s, s^{\prime} \in S_{\sigma, \eta}$, so that both (PastPA) and (ImFDis) hold for each of $s, s^{\prime}$, it suffices to show that the hypothesis $s<_{M} s^{\prime}$ leads to contradiction. Applying (ImFDis) (immediate future disagreement) to $s^{\prime}$, the hypothesis implies the existence of some $y_{0}$ between $s$ and $s^{\prime}$ (hence a $y_{0}$ such that $\left.y_{0}<_{M} s^{\prime}\right)$ such that $F\left(\sigma y_{0}\right) \neq F\left(\eta y_{0}\right)$. But now applying (PastPA) (past agreement) to $s^{\prime}$ yields $F\left(\sigma y_{0}\right)=F\left(\eta y_{0}\right)$. Contradiction.

Ad (iv): This is essentially the contrapositive of Definition 2 clause (1). For $F$ a proper property attribution on $\Sigma$ and $P$, if there is no splitting point for $\sigma, \eta$ prior to a point $x$, then $F(\sigma x)$ must be identical to $F(\eta x)$.

Ad (v): For reductio, assume $x \in R_{\sigma \eta} \cap R_{\eta \gamma}$ but $x \notin R_{\sigma \gamma}$. By Definition 3, the latter implies that we may choose $s$ such that $\left(s<x \wedge s \in S_{\sigma \gamma}\right)$, which in turn implies by (5), that is, by the immediate future disagreement clause of the definition of set $S_{\sigma \gamma}$ of splitting points, that we may find a $y$ such that

$$
s<y<x \wedge F(\sigma y) \neq F(\gamma y) .
$$

Regions of overlap are evidently closed downward, so that with our initial assumption, $y<_{M} x$ implies $y \in R_{\sigma \eta} \cap R_{\eta \gamma}$. By item (iv) of this Fact, $F(\sigma y)=F(\eta y)$ and $F(\eta y)=F(\gamma y)$, and hence $F(\sigma y)=F(\gamma y)$. Contradiction with $(\dagger)$.

Observe that in the proof above we used clause (1) but not clause (2) of Definition 2.

\subsection{Defining MBS's}

After this preliminary work, we turn now towards defining MBS's and showing that they satisfy the BST axioms. The first task is to find a correlate for the BST notion of Our World, i.e., a base set and an ordering. Given $\mathfrak{M}=\langle\Sigma, F, P\rangle$, following Müller (2002), we take the elements of a base set to be equivalence classes of a certain relation $\equiv_{R}$ on $\Sigma \times \mathbb{R}^{4}$. The idea is to "identify" points in regions of overlap; hence the relation $\equiv_{R}$ is defined as below. Müller proves $\equiv_{R}$ to be an equivalence relation on $\Sigma \times \mathbb{R}^{4}$. We will write $[\sigma x]$ for the equivalence class of $\sigma x$ with respect to the relation $\equiv_{R}$.

Definition 5 (MBS equivalence, MBS base set, and MBS ordering) Given $\mathfrak{M}=$ $\langle\Sigma, F, P\rangle$, the relation $\equiv_{R}$ on $\Sigma \times \mathbb{R}^{4}$ is defined as:

$$
\sigma x \equiv_{R} \eta y \text { iff } x=y \text { and } x \in R_{\sigma \eta} .
$$


We set

$$
[\sigma x]:=\left\{\eta x \mid \sigma x \equiv_{R} \eta x\right\} .
$$

The base set $B$ is defined by

$$
B:=\left\{[\sigma x] \mid \sigma \in \Sigma, x \in \mathbb{R}^{4}\right\} .
$$

The ordering $\leqslant_{R}$ on $B$ is defined by

$$
[\sigma x] \leqslant_{R}[\eta y] \text { iff } x \leqslant_{M} y \text { and } \sigma x \equiv_{R} \eta x .
$$

We will write $<_{R}$ for the strict counterpart of $\leqslant_{R}$.

As Müller shows, $\leqslant_{R}$ is a partial ordering on $B$. Note that if $x$ is not in the region $R_{\sigma \eta}$ of overlap of $\sigma$ and $\eta$, then $[\sigma x] \Varangle_{R}[\eta y]$ (for all $y$ ), but if $x$ is in $R_{\sigma \eta}$, for $[\sigma x] \leqslant_{R}[\sigma y]$ we need only check the Minkowski ordering, $x \leqslant_{M} y$.

Given $\mathfrak{M}=\langle\Sigma, F, P\rangle$, a natural notion of the course of events corresponding to scenario $\sigma$ is the set $\left\{[\sigma x] \mid x \in \mathbb{R}^{4}\right\}$ of equivalence classes. Knowing the set, that is, knowing each equivalence class from it, gives us all there is to be known about this course of events, that is, a property assignment for $\sigma$ and every $x \in \mathbb{R}^{4}$. This motivates defining $\left\{[\sigma x] \mid x \in \mathbb{R}^{4}\right\}$ as a "B-history."

Definition 6 (B-histories) Given $\mathfrak{M}=\langle\Sigma, F, P\rangle$, we define "the $B$-history corresponding to $\sigma \in \Sigma$," written $b_{\sigma}$, as $\left\{[\sigma x] \mid x \in \mathbb{R}^{4}\right\}$.

$B$-Hist is the set of all $B$-histories.

Given $\mathfrak{M}=\langle\Sigma, F, P\rangle$, our plan is to take the pair $\left\langle B, \leqslant_{R}\right\rangle$ to be an MBS representation of Our World. To this end we need a condition to assure an obviously desirable fact, namely that each $B$-history is identical to a maximal upward directed subset of $B$ (i.e., a history in the BST sense), and vice versa.

Condition 7 (the chain condition) Given $\mathfrak{M}=\langle\Sigma, F, P\rangle$, for a maximal upwarddirected subset $h$ of $B$ and $x \in \mathbb{R}^{4}$, define $\Sigma_{h}(x):=\{\sigma \in \Sigma \mid[\sigma x] \in h\}$.

For every maximal upward-directed set $h \subseteq B$ and for every chain $L \subseteq \mathbb{R}^{4}$, we require $\bigcap\left\{\Sigma_{h}(x) \mid x \in L\right\} \neq \emptyset$.

Given a maximal upward directed subset $h \subseteq B$ and $x \in \mathbb{R}^{4}$, we may ask for which $\sigma \in \Sigma$ the equivalence class $[\sigma x]$ belongs to $h . \Sigma_{h}(x)$ collects all such $\sigma \in \Sigma$. [For more information on the concept just defined, cf. Müller (2002); for a discussion of the chain condition, cf. Wroński and Placek (2009).]

Finally, we define MBS's in the present sense.

Definition $8(M B S)$ A triple $\mathfrak{M}=\langle\Sigma, F, P\rangle$ is an MBS iff $\Sigma$ is a non-empty set of scenarios, $F$ is a proper property attribution on $\Sigma$ and $P, P$ is a nonempty set of properties, and $\langle\Sigma, F, P\rangle$ satisfies the chain condition.

Here is a collection of small facts to which we appeal in our proofs, often silently. 
Fact 9 Let $\mathfrak{M}=\langle\Sigma, F, P\rangle$ be an MBS. Then:

(1) $[\sigma x] \leqslant_{R}[\sigma y]$ iff $x \leqslant_{M} y$;

(2) $[\eta x] \in b_{\sigma}$ iff $[\sigma x]=[\eta x]$ iff $\sigma x \equiv_{R} \eta x$ iff $x \in R_{\sigma \eta}$ iff $\neg \exists s\left(s \in S_{\sigma \eta} \wedge s<_{M} x\right)$.

We are now going to formulate our main theorem, which summarizes all the major information about MBS's, and states the desired facts mentioned before.

Theorem 10 Suppose that $\mathfrak{M}=\langle\Sigma, F, P\rangle$ is an MBS.

1. Every B-history is a maximal upward directed subset of $B$;

2. Every maximal upward directed subset of $B$ is a B-history;

3. To every $B$-history there corresponds a unique $\sigma \in \Sigma$, i.e., for every pair $b_{\sigma}, b_{\eta}$ of $B$-histories, $b_{\sigma}=b_{\eta}$ iff $\sigma=\eta$;

4. $\left\langle B, \leqslant_{R}\right\rangle$ as defined in Definition 5 satisfies the BST axioms described in Section 2.3;

5. For every pair $b_{\sigma}, b_{\eta}$ of $B$-histories, $[\sigma x]$ is maximal in $b_{\sigma} \cap b_{\eta}$ iff $x \in S_{\sigma \eta}$ (a perfect match between choice events and splitting points);

6. Every B-history is isomorphic to Minkowski spacetime;

$\mathfrak{M}$ permits the introduction of common st-locations;

Each B-history can be viewed as a differential manifold.

The clauses 1-3 can be summarized by saying that there is a perfect match between scenarios, $B$-histories, and maximal upward directed subsets of $B$.

Proof Ad 1: See the proof of Lemma 4 of Wroński and Placek (2009), which is a brushed-up version of the proof of "the first direction" of Lemma 3 of Müller (2002).

Ad 2: See the proof of Lemma 8 of Wroński and Placek (2009).

Ad 3: To the left, the proof is immediate. In the opposite direction, assume for reductio that (i) $b_{\sigma}=b_{\eta}$ and (ii) $\sigma \neq \eta$. By Fact 9(2), (i) is equivalent to $(\dagger) \forall z \in \mathbb{R}^{4}:([\sigma z]=[\eta z])$. (ii) entails that $S_{\sigma \eta} \neq \emptyset$-cf. Fact 4(i). Let us take $s_{0} \in S_{\sigma \eta}$ and $y$ such that $s_{0}<_{M} y$. By Fact 9(2): $[\sigma y] \neq[\eta y]$. Contradiction with $(\dagger)$.

Ad 4: Non-emptiness of $B$ immediately follows from non-emptiness of $\Sigma$, which is required by Definition 8 . To prove the prior choice principle, we need to show the following: ( $\dagger$ ) if $L$ is a lower bounded chain in $\left\langle B, \leqslant_{R}\right\rangle$ such that for some $\sigma, \eta \in \Sigma: L \subseteq b_{\sigma} / b_{\eta}$, then there is a maximal element $\left[\sigma s_{0}\right]$ of $b_{\sigma} \cap b_{\eta}$ such that $\forall[\sigma x] \in L:\left[\sigma s_{0}\right]<_{R}[\sigma x]$.

To prove this, let $L$ be as in the antecedent of $(\dagger)$. Let us define $E:=\{x \in$ $\left.\mathbb{R}^{4} \mid[\sigma x] \in L\right\}$. Since $[\sigma x] \leqslant_{R}[\sigma y]$ iff $x \leqslant_{M} y, E$ is a lower bounded chain in $\left\langle\mathbb{R}^{4}, \leqslant_{M}\right\rangle$. From the antecedent of $(\dagger)$, for an arbitrary $[\sigma x] \in L$ : $[\sigma x] \notin b_{\eta}$, which by Fact 9(2) is equivalent to $\exists s\left(s \in S_{\sigma \eta} \wedge s<x\right)$. Accordingly, $E$ satisfies the antecedent of clause (2) of Definition 2. By this clause, $\exists s_{0}\left(s_{0} \in S_{\sigma \eta} \wedge \forall x\left(x \in E \rightarrow s_{0}<x\right)\right)$. Clearly, $s_{0} \in R_{\sigma \eta}$, so by Fact 9 (2) $\left[\sigma s_{0}\right] \in b_{\eta}$ and hence $\left[\sigma s_{0}\right] \in b_{\sigma} \cap b_{\eta}$. Moreover, ( $\left.\ddagger\right)\left[\sigma s_{0}\right]$ is maximal in $b_{\sigma} \cap b_{\eta}$. For, for any $[\sigma y]$, if $\left[\sigma s_{0}\right]<_{R}[\sigma y]$, then $s_{0}<_{M} y$ and hence by the same Fact, $[\sigma y] \notin b_{\eta}$ and hence $[\sigma y] \notin b_{\sigma} \cap b_{\eta}$. Finally, since $s_{0}<_{M} x$ for 
every $x \in E, \forall[\sigma x] \in L:\left[\sigma s_{0}\right]<_{R}[\sigma x]$, which taken together with ( the consequent of $(\dagger)$.

For the proof of the other axioms of BST, consult Müller's (2002) proof of his Theorem $1 .{ }^{17}$

Ad 5: To the left first: Let $x \in S_{\sigma \eta}$. Then $x \in R_{\sigma \eta}$, which by Fact 9(2) is equivalent to $[\sigma x] \in b_{\eta}$, which entails $[\sigma x] \in b_{\sigma} \cap b_{\eta}$. To show that $[\sigma x]$ is maximal in $b_{\sigma} \cap b_{\eta}$, note that if for some $[\sigma y],[\sigma x]<_{R}[\sigma y]$, then $x<y$, and hence

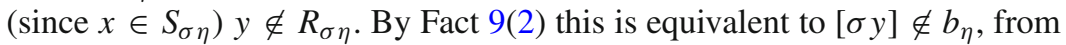
which $[\sigma y] \notin b_{\sigma} \cap b_{\eta}$ follows. Accordingly, $[\sigma x]$ is maximal in $b_{\sigma} \cap b_{\eta}$.

To the right: Let $(\$)[\sigma x]$ be maximal in $b_{\sigma} \cap b_{\eta}$. Consider a chain $E$ in $\left\langle\mathbb{R}^{4}, \leqslant_{R}\right\rangle$ such that $x$ is its proper infimum. It follows that $(\star) \forall y \in E$ : $[\sigma y] \notin b_{\sigma} \cap b_{\eta}$. By Fact $9(2)(\star)$ is equivalent to $\forall y \in E \exists s\left(s \in S_{\sigma \eta} \wedge s<y\right)$. Accordingly, $E$ satisfies the antecedent of clause (2) of Definition 2. By this clause, there is $s_{0}$ such that $(\dagger) s_{0} \in S_{\sigma \eta}$ and $(\dagger) \forall y\left(y \in E \rightarrow s_{0}<y\right)$. Since $x$ is the (proper) infimum of $E,(\ddagger)$ implies that $s_{0} \leqslant_{M} x$. But it cannot be that $s_{0}<_{M} x$ since given $(\dagger), s_{0}<_{M} x$ is equivalent (by Fact 9(2)) to $[\sigma x] \notin b_{\eta}$, which contradicts $(\$)$. Hence $x=s_{0}$, and by $(\dagger), x \in S_{\sigma \eta}$.

Ad 6: Function $f$ from $b_{\sigma} \in B$-Hist onto $\mathbb{R}^{4}$, defined by: $f([\sigma x])=x$, provides the sought-for isomorphism. By the definition of $B$-histories $f$ is indeed onto $\mathbb{R}^{4}$. It is also immediate to see that $[\sigma x] \leqslant_{R}[\sigma y]$ iff $x \leqslant_{M} y-\mathrm{cf}$. Definition 5 . Next, the set $\{[\eta x] \mid \eta \in \Sigma\}$ is the st-location of event $[\sigma x] \in B$-it is straightforward to see that sets having this form satisfy conditions (1) and (2) on st-locations of Sect. 2.7.

Finally, each $B$-history can be viewed as a differential manifold since for each $B$-history the function $f$ (introduced above) serves as its global chart. ${ }^{18}$

\subsection{Taking stock}

Let us take stock of this construction of MBS's. The first message is that histories in MBS's are isomorphic to Minkowski spacetime. Accordingly, up to isomorphism, a history of an MBS is a spacetime (Minkowski spacetime).

The second message, or rather observation, is how delicate the construction is with respect to theories of modern physics. Even if the spatiotemporal structure of our Universe were adequately represented by Minkowski spacetime (which it is not), a few conditions must be satisfied, on the part of physics, for our construction of MBS's to go through. First, the physical description must come in the form of a property attribution to spatiotemporal points. Second, the property attribution must be quite specific; we here required it to be "proper."19 Finally, an additional condition,

\footnotetext{
17 In his proof, Lemma 3 requires finitistic assumptions. A non-finitistic version of this lemma is proved in Wroński and Placek (2009) as Lemma 8, using the chain condition.

${ }^{18}$ For manifold theory, see e.g., Hall (2004).

19 This is not to say that some other condition on property attributions and a matching definition of splitting points would not do the job. The point is that this notion must be somehow regimented to be of use in producing BST models.
} 
like our Chain Condition 7 or Müller's finitistic condition, must hold to guarantee isomorphism between $B$-histories and Minkowski spacetime. Yet, with mathematical necessity, if these conditions hold, the resulting MBS has sets of choice events (in an extreme one-history case, there is, namely, the empty set of choice events), with any two histories overlapping in a region delineated from above by a figure similar to a generalized letter W. This derivation is relevant to Earman's complaint quoted in Sect. 5 that modern physics does not know of this kind of pattern of choice events.

\subsection{Historical remarks}

Our construction of MBS's diverges from the constructions of Müller (2002) and Wroński and Placek (2009) because it aims to be more physics-oriented. The authors mentioned begin their work with specifying a set $\Sigma$ of labels for scenarios and a collection $\left\{S_{\sigma, \eta}\right\}_{\sigma, \eta \in \Sigma}$ of sets of splitting points, where each $S_{\sigma \eta}$ possesses properties listed in Fact 4(i)-(iv). Given the two primitive notions, that is, labels for scenarios and sets of splitting points, they define MBS's and show, on the assumption of certain additional conditions, that MBS's satisfy BST axioms. The authors diverge over these additional conditions: Müller assumes finitistic requirements whereas Wroński and Placek accepts a "topological" postulate that is equivalent to the chain condition. The difference notwithstanding, an MBS model is, in their sense, a pair $\left\langle\Sigma,\left\{S_{\sigma, \eta}\right\}_{\sigma, \eta \in \Sigma}\right\rangle$. In contrast, our point of departure is a property attribution to points in scenarios. Accordingly, an MBS model is, in our sense, a triple $\langle\Sigma, F, P\rangle-\mathrm{cf}$. Definition 8. Splitting points are then a derived notion-see Definition 1 and, as Fact 4(i)-(iv) shows, they satisfy the conditions assumed by Müller and Wroński and Placek. Accordingly, given that $\mathfrak{M}=\langle\Sigma, F, P\rangle$ is an MBS in the sense of Definition $8,\left\langle\Sigma,\left\{S_{\sigma, \eta}\right\}_{\sigma, \eta \in \Sigma}\right\rangle$ with $\left\{S_{\sigma, \eta}\right\}_{\sigma, \eta \in \Sigma}$ defined by Definition 1 is an MBS in the sense of Wroński and Placek (2009). If finitistic assumptions concerning sets $S_{\sigma \eta}$ are assumed, $\left\langle\Sigma,\left\{S_{\sigma, \eta}\right\}_{\sigma, \eta \in \Sigma}\right\rangle$ is an MBS in the sense of Müller (2002).

The need for the mentioned additional conditions (the chain condition or finitistic assumptions) stems from the desire to identify $B$-histories with histories in the BST sense.

\section{Further replies to Earman}

\subsection{BST: Hausdorff property}

Earman argues that "literal branching of a relativistic spacetime" (p. 193) can lead to a failure of the Hausdorff property. In turn, the failure of the Hausdorff property has various negative consequences for physics, which he lists. So, in order to avoid these consequences, an individual spacetime in BST had better satisfy the Hausdorff property. We first ask if each BST history (or spacetime) satisfies the Hausdorff property. There is also a larger question, going beyond Earman's worry, of whether each BST model satisfies the Hausdorff property. Although we pose these questions quite generally (about BST models), our results obviously apply to MBS's. 
Let us recall the Hausdorff property:

Definition 11 Suppose that $\mathfrak{T}$ is a topology on set $X$. Then $\mathfrak{T}$ has the Hausdorff property iff for any two distinct $e, e^{\prime} \in X$ there are disjoint sets $U, V \in \mathfrak{T}$ such that $e \in U$ and $e^{\prime} \in V$.

To investigate the Hausdorff property, however, we need a topology for BST. We introduce below, following Bartha, what we claim to be a thoroughly natural topology for BST (generally), and for MBS's in particular. ${ }^{20}$

Definition 12 (time-or-light-like paths and diamonds) Let $O W=\langle W, \leqslant\rangle$ be a BST model. $t$ is a time-or-light-like path $(t \in T T L)$ iff $t$ is a maximal chain in $\langle W, \leqslant\rangle$.

Given a time-or-light-like path $t \in T T L$, we define

$$
d_{t}^{e_{1} e_{2}}:=\left\{y \in W \mid e_{1}<e_{2} \wedge\left\{e_{1}, e_{2}\right\} \subseteq t \wedge e_{1} \leqslant y \leqslant e_{2}\right\} .
$$

We call $d_{t}^{e_{1} e_{2}}$ "the diamond oriented by $t$ with vertices $e_{1}$ and $e_{2}$."

Definition 13 (the diamond topology on $W) Z$ is an open subset of $W, Z \in \mathfrak{T}(W)$, iff $Z=W$ or for every $e \in Z$ and for every $t \in T T L$ containing $e$ there is a diamond $d_{t}^{e_{1} e_{2}} \subseteq Z$ that is oriented by $t$ with $e$ strictly between the diamond's vertices $e_{1}$ and $e_{2}$.

Thus, $Z \in \mathfrak{T}(W)$ iff $Z=W$ or

$$
\forall e \in Z \forall t \in T T L\left(e \in t \rightarrow \exists e_{1}, e_{2} \in t\left(e_{1}<e<e_{2} \wedge d_{t}^{e_{1} e_{2}} \subseteq Z\right)\right)
$$

The condition of the above definition can be used to define the topology $\mathfrak{T}(h)$ on history $h \in$ Hist as well:

$Z \in \mathfrak{T}(h)$ iff $Z=h$ or

$$
\forall e \in Z \forall t \in T T L\left((t \subseteq h \wedge e \in t) \rightarrow \exists e_{1}, e_{2} \in t\left(e_{1}<e<e_{2} \wedge d_{t}^{e_{1} e_{2}} \subseteq Z\right)\right)
$$

It is straightforward to see that the family $\mathfrak{T}$ of open sets, as defined above, forms a topology. This means that $\emptyset \in \mathfrak{T}, W \in \mathfrak{T}$, if $U, V \in \mathfrak{T}$, then $U \cap W \in \mathfrak{T}$, and the union of a family of sets from $\mathfrak{T}$ belongs to $\mathfrak{T}$.

We have now these two theorems, highly relevant to Earman's concern with the Hausdorff property.

Theorem 14 Let $h$ be a history of a BST model $\mathcal{W}$. Then the Hausdorff property is satisfied in $h$ (in the topology $\mathfrak{T}(h)$ ).

To present our next theorem, we need to introduce a particular feature of BST, known as "indeterminism without choice" (cf. BST 1992). It could perhaps be best characterized by considering various ways in which two paths $t_{1}, t_{2} \in T T L$ can form a $Y$-shaped fork.

20 Cf. "postprint" to Belnap (1992), footnote 26. As for a topology being natural, this should be judged by whether or not it can yield open subsets isomorphic to open subsets in the standard topology of $\mathbb{R}^{N}$. Our topology has this feature, if applied to MBS's. 
Definition 15 (Y-fork) $t_{1}, t_{2} \in T T L$ form a Y-fork iff

1. $t_{1} \neq t_{2}$,

2. $t_{1} \cap t_{2} \neq \emptyset$,

3. $\forall x \in t_{1} \cup t_{2}\left(x \in t_{1} \cap t_{2} \vee\left(t_{1} \cap t_{2}<x \wedge\left(x \in t_{1} / t_{2} \vee x \in t_{2} / t_{1}\right)\right)\right)$.

Every Y-fork has its trunk $t_{1} \cap t_{2}$, and two arms $t_{1} / t_{2}$ and $t_{2} / t_{1}$. A Y-fork may be entirely a spatio-temporal matter. In order to characterize Y-forks that exhibit indeterminism, we define a "modal fork" as follows.

Definition 16 (modal-fork) $t_{1}, t_{2} \in T T L$ form a modal fork iff they form a Y-fork and $\exists h_{1}, h_{2} \in$ Hist $t_{1} / t_{2} \subseteq h_{1} / h_{2} \wedge t_{2} / t_{1} \subseteq h_{2} / h_{1}$.

In the presence of Definition 15, the condition on modal forks is equivalent to this claim:

$$
\forall x \in t_{1} \cup t_{2}\left(t_{1} \cap t_{2}<x \rightarrow x \in h_{1} / h_{2} \vee x \in h_{2} / h_{1}\right)
$$

Given an Y-fork, its trunk might have a (unique) maximum, or not. For a modal fork, it is evident that if $t_{1} \cap t_{2}$ has a maximum, then it is a choice event for some two histories; otherwise we have indeterminism without choice:

Definition 17 (indeterminism without choice) A modal fork exhibits indeterminism without choice in case its trunk has no maximum.

The upshot of these definitions is a three-fold classification of Y-forks. A Y-fork may be modal or not, and if is modal, it either exhibits indeterminism without choice, or not. Note that the essence of indeterminism without choice is not a matter of lacking a choice event (this is impossible by the prior choice principle of BST), but that every choice event is external to a modal fork in question. The lemma that follows the fact below shows a crucial feature of indeterminism without choice:

Fact 18 The trunk of a Y-fork is upper bounded by an element of each of $t_{1} / t_{2}$ and $t_{2} / t_{1}$.

Proof By Definition 15(1) and maximality of $t_{1}, t_{2}$, there is $x \in t_{1} / t_{2}$, so $x \notin t_{1} \cap t_{2}$, so $t_{1} \cap t_{2}<x$ by Definition 15(3); and similarly for $t_{2} / t_{1}$.

Lemma 19 Let a modal fork exhibit indeterminism without choice. Then the trunk of this fork has two distinct history-relative suprema. That is, if $t_{1}, t_{2} \in T T L$ form a modal fork and $t_{1} \cap t_{2}$ has no maximum, then $\sup _{h_{1}}\left(t_{1} \cap t_{2}\right) \neq \sup _{h_{2}}\left(t_{1} \cap t_{2}\right)$ for some $h_{1}, h_{2} \in$ Hist.

Proof For brevity, define $\Gamma=\left(t_{1} \cap t_{2}\right), \gamma_{1}=\sup _{h_{1}}(\Gamma), \Delta_{1}=\left(t_{1} / t_{2}\right)$, and similarly for $\gamma_{2}, \Delta_{2}$. We show that $\gamma_{1} \neq \gamma_{2}$.

Note that $\left(\Gamma \cup \Delta_{1}\right)=t_{1}$ by calculation, $\Gamma \neq \varnothing$ by Definition $15(2), \Delta_{1} \neq \varnothing$ by Fact 18, and $\Gamma<\Delta_{1}$ by Definition 15(3). By Definition 16, $\Delta_{1} \subseteq h_{1} / h_{2}$, so $\Gamma \subseteq h_{1}$ by downward closure of histories, so $\gamma_{1}$ exists (by the BST axiom of history-relative suprema) and $\Gamma \leqslant \gamma_{1} \leqslant \Delta_{1}$. Since $\left(\Gamma \cup \Delta_{1}\right)=t_{1}$, density and maximality of $t_{1}$ imply that $\gamma_{1} \in\left(\Gamma \cup \Delta_{1}\right)$. If $\gamma_{1} \in \Gamma$, then $\gamma_{1}$ would be maximum in $\Gamma$, violating the assumption of indeterminism without choice (Definition 17). So $\gamma_{1} \in \Delta_{1}$. An exactly parallel argument yields $\gamma_{2} \in \Delta_{2}$. Since $\left(\Delta_{1} \cap \Delta_{2}\right)=\emptyset, \gamma_{1} \neq \gamma_{2}$. 
We are now ready to state our second theorem concerning the Hausdorff property.

Theorem 20 Let $\mathcal{W}$ be a BST model. If some modal fork in $\mathcal{W}$ exhibits indeterminism without choice, then the Hausdorff property fails in $\mathcal{W}$ (in the topology $\mathfrak{T}(W)$ ).

We omit the proofs of Theorems 14 and 20.

What is the significance of these theorems? As Pruning argues, a failure of the Hausdorff property in an individual spacetime has severe consequences. Against the worry that these consequences concern BST, our Theorem 14 gives the reassuring "don't worry, be happy with BST", since each BST spacetime/history has the Hausdorff property.

Our Theorem 20 concerns a larger issue of whether a BST model, as a whole, has the Hausdorff property. Since (to recall) a BST model aims to represent indeterminism, the issue is analogous to asking, in the framework of ensemble branching, whether an ensemble of spacetimes has the Hausdorff property. Of course, for the latter question to be tractable, one needs to know a topology on a set of GR spacetimes, not merely on a given GR spacetime. The theorem ties together a failure of the Hausdorff property and indeterminism without choice, the latter being an important feature of BST. That is, although there are BST models without this feature, they either fail to represent spatial aspects, or involve strange massive modal correlations. ${ }^{21}$

Indeterminism without choice is familiar to both the experimenter and the layman, as this schematic story attempts to illustrate. In order for a given object to reach a detector and be detected there, it needs to pass through a shutter, which operates indeterministically: if an $\alpha$-particle decays in its vicinity, the shutter opens; otherwise it remains closed. Now, as for the question: "why has our object been detected?", the (partial) answer is: "Because the $\alpha$-particle decayed in the past of the detection," which nicely reflects the meaning of the prior choice principle. Since the decay was external to the object's trajectory, it also makes sense to ask: "Which part of the object's trajectory is in the causal future of the decay, and which part is not?" This second question underwrites indeterminism without choice. Posing this question is natural if indeterminism reigns in a spatially extended world.

These subtleties aside, branching, whether Hausdorff or non-Hausdorff, entails bifurcating time-like paths that seem to trouble Earman:

But how would such a particle know which branch of a bifurcating geodesic to follow? This problem has led general relativists to shun non-Hausdorff spacetimes that involve non-Hausdorff branching [...]

The worry is groundless, as it presupposes the mistaken doctrine of the actual future. To use (with reluctance) the epistemic language of the physicists, the faulty presupposition of the particle's ignorance of which way to go is that there is one selected possible future (the actual future) among many possible futures, so that the particle does not know which of the possible futures is actual (see FF Sect. 2B.10). The next

\footnotetext{
21 A relevant fact is this: Let $W$ be a BST model, in which every modal fork has a trunk with a maximum. Moreover, let there exist a maximum $m$ of the trunk of a modal fork and $e \in W$ such that $m$ SLR $e$. Then there is modal funny business in W. (For the notion of modal funny business, cf. Müller et al. (2008)).
} 
section details our complaints about this mistake. As for bifurcating paths, we say: If the bifurcation is modal, that's just indeterminism.

On a related issue, Earman opines that "[i]f non-Hausdorff [...] branching is to be taken seriously, what is needed is a physical theory that prescribes the dynamics of branching" (p. 202). Leaving the qualification"seriously" aside, we readily agree that there is much to be done to make contact between BST and physics. At present, we know how to link standard quantum mechanics (in the consistent histories formulation) to BT, ${ }^{22}$ and Minkowski spacetime to BST. The issue of supplying dynamics for branching histories, and in particular, relating BST to general relativity, is largely a white area.

\subsection{The thin red line}

The "thin red line" (TRL) theory is the colorful term of Belnap and Green (1994) and FF for the theory that, in Earman's words, "as of an indeterministic moment ("branch point'), exactly one future reaching branch is the actual future" (p. 190). Earman seems to endorse as respectable the heavily metaphysical view "that (as of now) there is [a] fact of the matter as to which of the possible futures is the actual future" (p. 190). ${ }^{23}$

The TRL is not, we think, respectable. It is a muddled theory that tries to be both deterministic and indeterministic. According to FF, if determinism be true, then there is only one "possible future reaching branch," period. But if some moment is indeterministic, admitting many possible "future reaching branches," then, argues FF, it is truly a muddle to suppose that one of these is "actual," and the others "merely" possible - even though the supposition implies that others have no possibility of being actual. As New Englanders say, "Fish or cut bait." FF devotes an entire chapter [which is essentially grounded in Belnap and Green (1994)] to giving reasons for the "no TRL" view. ${ }^{24}$ In some minds (not ours), the matter remains, however, debatable; see Øhrstrøm (2009) for the most recent and best defense known to us of the contrary view. ${ }^{25}$ Moving to the central point, we agree with Pruning (p. 190) that the denial of the TRL theory is logically independent of BT or BST92, and in fact it is evident by inspection that such a denial is no part of the axiomatization of BT or BST92. ${ }^{26}$ Pruning (p. 192) correctly says, "To get that result some additional piece of metaphysics would have to be added," (p. 190) a step that is rightly characterized as "something beyond ensemble branching." What Pruning fails to admit, however, is that it is equally

\footnotetext{
22 Cf. Müller (2007). Since quantum mechanics is non-relativistic, it can be analyzed in BT, but not in BST, which is a relativistic framework.

${ }^{23}$ In order to represent Earman's opinion by quotation, we extracted the quote from a sentence beginning "It certainly does not follow that (as of now) there is no fact of the matter ...".

${ }^{24}$ See also Perloff and Belnap (2010).

25 The most recent defenses are A. Malpass's talk at the conference "Indeterminacy and Branching Time", Bristol 2010 and J. Wawer's presentation at the "Causes and Tenses" workshop, Kraków 2010.

26 Pruning describes FF as using indeterminism "in a sense that validates the no-thin-red-line doctrine, a future-branching tree structure for time and spacetime, and the other postulates imposed on this structure." This is essentially correct, although (regrettably) it seems falsely to suggest that no-TRL is a postulate of BT or BST92.
} 
a step beyond branching either in the ensemble sense or the BST sense to assert that there is a "fact of the matter as to which of the possible futures is the actual future" (p. 190). If no-TRL is a piece of unphysical metaphysics, then so is the TRL doctrine that Pruning seems to espouse.

In its footnote 10, Pruning explicitly denies the symmetry we claim: MacFarlane (2003), who does not hold with the TRL, is described as attributing to those who do "a 'posit' of a metaphysical entity - the thin red line." Earman continues, "On the contrary, it is those deniers of the thin red line-like MacFarlane and the Belnap school—who hold that future tensed but not past tensed statements may lack a determinate truth value that need to rely on some posit beyond indeterminism in the ensemble branching sense to motivate their semantic rules" (p. 192). Earman does not support this claim, and indeed it is demonstrably false. A straightforward examination of the semantics of indeterminism as given by MacFarlane, ${ }^{27}$ or of the detailed formal semantics as given in any of FF or the various papers by Belnap, Placek, or Müller that are referenced by Pruning (as well as the additional papers referenced here), reveals these authors do not claim that the TRL is inconsistent. Rather, these papers make a point of illuminating uses of the future tense in terms of a semantics that does not refer to a TRL, and that furthermore would not be improved were the semantics to make such a reference.

\subsection{Semantic rule $(R)$}

Pruning considers equating the no-TRL doctrine with a "semantic rule $(R)$," which is a wholly inadequate version of the supervaluation account of truth for future-tensed statements due to Thomason (1970) (an attribution not mentioned by Pruning), according to which a statement such as "There will be a sea battle" as uttered now may be true, false, or indeterminate depending on whether all, none, or some but not all futurereaching branches contain a "token" of the event-type 'sea battle.' We say "inadequate" because (1) obviously the account is only relevant to a small sample of future-tensed statements, and (2) it does not live up to the standards for semantic rules and theories inherited from Tarski: it cannot be counted as "formally correct," even for the few sentences to which it applies. For one thing, Pruning fails to refer to any theory of event types and event tokens.

In the same paragraph, Pruning declares that "a natural rule for assigning truth to future tensed statements" is a version of $(R)$ that "makes 'There will be a sea-battle' true in case the (hypothesized) unique actual future contains a sea-battle and false otherwise" (p. 191, our italics). We are unable to discern the difference between this version of $(R)$ and the TRL (Sect. 4.2). We note that Pruning makes no effort to give a scientific meaning to "actual future," and we remark again that we think that it cannot be done.

The paragraph continues with yet a third reading of $(R)$, "yielding a version of presentism on which contingent statements about the past as well as the future lack truth values" (p. 191). The symmetry to which Pruning refers here is mentioned again

\footnotetext{
27 Pruning explicitly declines to consider this theory.
} 
in its footnote 10 , where, to the extent that we understand it, the question of a distinguished possible history through a moment or event is conflated with the question of past/future asymmetry. Neither denying the asymmetry, as Earman does, nor affirming it, as we do, is relevant to the existence or nonexistence of a privileged "actual" history (a TRL). See Sect. 4.4 for the quite separate asymmetry question.

Our central argument against the TRL — the history containing the"actual future"is this: Positing a TRL does no work in understanding statements involving a reference to future happenings, whether commonsensical or in the language of physics, and indeed tends to interfere with that understanding. Pruning is misleading to the extent that it suggests the contrary.

Example. Assuming indeterminism, I bet you a dollar that the coin will come up heads. A naive explanation of bets has it that I am betting that the coin will come up heads in the actual future - the one that, at the moment of betting, is fixed as uniquely privileged - regardless of what happens in alternative physically possible futures. Consequently, on the naive reading, we bet on what is already actually fixed, even if we don't know which future is the one that will actually happen. (The indeterminism is merely epistemic.)

In many cases we take our bets to have indeterministic context: in such cases we believe that a thing we bet on is not settled, and not merely unknown. Provided the aim is an analysis of betting-on-heads in an indeterministic context, the "regardless" approach is just wrong as it yields an inadequate analysis. Besides, the very essence of this approach, which is a gesture towards singling out one (actual) history, is hopeless: the singling-out cannot be accomplished by no matter how idealized the agent.

As a start of a subtler analysis, one specifies all possible historical continuations of a particular bet on a particular coin toss coming up heads: If we end up in one of those possible continuations in which the coin comes up heads, I win and you owe me one dollar; and of equal importance, if the history is one of those in which the coin does not come up heads, you win and I owe you one dollar. This essential and complete and entirely adequate explanation of our bet quantifies evenly over all histories that include the act of betting on the coin toss. It has no need of promoting one of the histories from "possible" to "actual." For a bet having the form "I bet on the truth of 'The coin will come up heads'," one needs a delicate account using "double time references" as in Belnap (2002a). Neither this account nor any other useful account of betting appeals to "the actual future" (to the TRL).

Example. Assuming indeterminism, and finding from physical analysis that the odds are in my favor (say ten to one), I predict that this particular measurement of the electron will yield "spin up." Faulty explanation of the prediction: I am predicting that the outcome of the measurement will be "spin up" on the actual history, regardless of what happens in alternative physically possible histories. Again, the "regardless" clause is wrong, and in just the same way. To explain the act of prediction, you must tell of its consequences on each member of an ensemble of physically possible histories that includes the act of predicting the outcome of a particular measurement, whether the outcome is favorable or not.

The lesson is even clearer if, instead of predicting, I just make a ten-to-one claim concerning the chances of "spin up" or not. It doesn't help at all to say that I am giving the chances of "spin-up" on the actual history, regardless of what happens on merely 
possible histories. You can say it, and say it consistently, and even with heavy breathing, but it remains mere verbiage. The probability claim characterizes the ensemble of future developments from an initial occasion or event; it does not characterize a single history, actual or not. See especially Müller (2005).

We grant that not only everyday folks, but highly trained scientists, are inclined to use the language of "actuality" to contrast with "physical possibility." But that is only because their training doesn't extend to the relevant portions of the philosophy of language. Since Kaplan (1989), it has been clear to philosophers (if not physicists) that the best careful uses of "actual" need to be grounded in something like "what is settled true at the occasion of utterance." 28

The issue of whether "Spin-up will come to pass" and "Spin-up will actually come to pass" are equivalent sentences is subtle. It suffices here to say that if these sentences are interpreted as "stand-alone," assuming a natural syntax and semantics for "actually" they are indeed equivalent. ${ }^{29}$

\subsection{Past/future asymmetry}

BT and BST treat the past and future asymmetrically by means of the postulate of "no backward branching" of BT and the prior choice principle of BST. Pruning ignores the arguments of FF in favor of no backward branching, and equally ignores the crucial and detailed role played by these postulates, especially in the technical analysis of causae causantes, or originating causes (Belnap 2005b). Instead, Pruning opines in an offhand fashion (pp. 191-192) that in virtue of laws that are time-reversal invariant, such asymmetry is untrue to physics as represented by ensemble branching. FF quotes the teaching of a distinguished physicist in its defense of no backward branching:

I want to suggest here that —once one accepts indeterminism - there is no reason against including irreversibility as part of the fundamental laws of nature... The realization of a specific pattern of new events, the selection of facts from possibilities is spontaneous and-apart from conservation laws-is governed by probability. It should be stressed that this picture does not touch CTP-invariance or detailed balancing. As Lüders has pointed out the term "time reversal" should be replaced by "motion reversal." One does not change the arrow of time but compares states with mirrored velocities (Haag 1990, p. 247).

Indeterminism brings in a distinction between the settled past and present and the open future. Haag suggests taking this asymmetry (which he somewhat confusingly calls irreversibility) to be a part of the fundamental laws of physics. Superficially, CPT invariance is in conflict with this asymmetry. We say "superficially" since the

\footnotetext{
28 MacFarlane (2003) argues for taking into consideration also an occasion of assessment.

29 For a distinction between stand-alone sentences and embedded sentences, see FF. Tenses and actuality are subtle matters, and cannot be treated with vigorous informal armwaving such as that employed by Pruning. To omit reference to the formal semantics given in the relevant publications is analogous to discussing general relativity without the language of differential manifolds, something that would properly scandalize Earman.
} 
invariance concerns what Haag and Lüders call "motion reversal" of states rather than the past-future asymmetry of events.

BT/BST concern events, not states, and in general both the theories are silent about the latter notion. In a particular class of BST models, MBS's, states can be easily introduced, however. The state of event $[\sigma x]$ is $F(\sigma x)$, i.e., a set of point properties (like values of physical fields). In MBS's states are repeatable, like states of physical theories, or common-sensical states (e.g., a body's temperature). In contrast, a particular event is not repeatable. Clearly, the motion reversal operates on states, not particular events.

There is no trouble in an MBS history having two intervals (i.e., dense chains) of events, a sequence of states assigned to one interval being the reverse of the sequence of states assigned to the other interval. There is also no trouble in there being two histories, each containing an interval of events, with the same sequence of states assigned, but each preceded by an event with a different state. Such sequences of states that fork towards the past seems to be a BST representation of the motion-reverse of two evolutions forking towards the future.

These non-problematic cases are local, however, as the relevant states are states of some segment(s) of histories. In contrast, we may consider the motion reversal applied to states of the whole history. A toy-model suggested to us by B. Roberts illustrates the point (the story is non-relativistic, so we talk of a BT model and pretend it is analogous to an MBS):

Let one spacetime have a ball spinning clockwise at a constant rate, for all time. Let another spacetime have the same spinning ball coming to a stop at some time $t$, after which it remains at rest forever. The motion-reverse of the first spacetime is a spacetime with the ball spinning counter-clockwise for all time and the motion-reverse of the second spacetime is one in which the ball is at rest until a time $t$, when it begins to spin counter-clockwise. Can the two motion-reversed spacetimes be interpreted as histories of a BT model?

If we keep the ordering $\leqslant$ fixed, that is, assume that before an event at time $t$ the ball spins in one spacetime and is at rest in the other, the two spacetimes cannot be accommodated in any BT model similar to MBS's. The MBS's postulate of proper property attribution requires that for every two histories there is a location (element of $\mathbb{R}^{4}$ ), below which the histories are qualitatively alike. But, since before $t$, in one spacetime the ball is spinning whereas in the other it is at rest, the spacetimes are not qualitatively alike below any $x \in \mathbb{R}^{4}$.

We find this a welcome fact that these two evolutions cannot be described by a BST model. No sense can be made of two alternative possible evolutions, separate before some event and combining into a single evolution after it. ${ }^{30} \mathrm{CPT}$ symmetry should not be interpreted as providing evidence for such evolution. CPT is a claim about models of a physical theory. It requires, in particular, that if a theory has two models exhibiting states forking towards the future, then the theory has as well some two models exhibiting states forking towards the past. The (mathematical) existence of such mathematical models should not be interpreted as suggesting a possibility of

30 For a detailed defense of the view that no particular event can be preceded by two alternative courses of events, in the context of no backward branching and the theory of agency, cf. FF Sect. 7A.2. 
a particular event (or a particular stage of a system) that is preceded by alternative evolutions. For more on why (we think) it is wrong to characterize indeterminism in terms of models of theories, see the next section.

\section{Indeterminism}

\section{Earman claims that}

[t]he postulates used by the Belnap program seem to place a priori constraints on physics. For example, members of the Belnap school speak of "choice points," which are to be thought of as the loci of indeterministic influences that radiate outwards along or within the future null cones of these loci. On this way of conceptualizing indeterminism, the members of the ensemble of physically possible models will typically agree at all points except those that lie on or inside the future null cones of the choice points. I know of no theory in modern physics which will produce this kind of indeterminism. Relativistic field theories, whether classical or quantum, typically entail lawlike connections among relatively spacelike events (p. 192). ${ }^{31}$

To respond, a particular class of BST models, MBS's, that we here developed, assumes a specific pattern of qualitative divergence, which we think is intuitive and weak (recall the past agreement and the immediate future disagreement). Nevertheless, as Earman says, no known physical theory exhibits this MBS-style pattern of qualitative differences. BT, however, can be used to model nonrelativistic quantum mechanics, as shown by Müller (2007); since this theory is non-relativistic, the analysis requires BT models, not BST models. ${ }^{32}$ Another possible area of application for BT is Norton's (2008) dome; since this is a system of classical mechanics, it requires BT, not BST. ${ }^{33}$

Given the weakness of assumptions underlying our construction of MBS's, we tend to say that this situation indicates that there is no indeterminism in the known relativistic theories, though, on the other hand, nonrelativistic quantum mechanics is a good candidate for an indeterministic theory. This moral is in sharp conflict with findings of Earman (1986) and Earman (2007), and this conflict calls for the re-assessment of the project of investigating indeterminism of physical theories. Our contention is that

\footnotetext{
31 The final sentence of this quotation from Earman suggests that BST cannot represent lawlike connections between space-like separated events, which is simply false. BST explicitly leaves room for what it calls "funny business," which is precisely (non-accidental) correlations between SLR events, devoting three publications entirely to their study. See Belnap (2002b, 2003b), and Müller et al. (2008) and Placek and Wroński (2009). Pruning cites these papers without considering them. (Pruning's reference to Belnap (2003b) mistakenly references Synthese rather than Philosophical studies).

32 Müller analyzes the consistent histories formulation of quantum mechanics.

33 As explained in Sect. 4.4, the motion-reversed trajectories and the motion non-reversed trajectories should not be represented in one BT model (we owe this point to Roberts). For a penetrating discussion on this subject we would like to thank the audience of TP's talk in February, 2010, at the Center for Philosophy of Science of the University of Pittsburgh, and Norton in particular.
} 
ensemble branching does not adequately represent (in)determinism. The usual intuitive notion of future-oriented indeterminism is phrased in terms of open possibilities: the world is indeterministic in some region (or at some stage, or at an event) if that region has more than one possible continuation. Clearly, the notion is modal, as it appeals to open possibilities. The notion of open future possibilities was banned from analytic philosophy because no one knew how to talk soberly about the open future. For this reason, many philosophers attempted to characterize (in)determinism in epistemic terms, like predictability or calculability. A locus classicus of this movement is Popper's (1982) book. This tendency was further reinforced by taking Laplace's vision as a starting point of a debate over (in)determinism: Laplace's demon was explained in epistemic terms. To the demon "nothing would be uncertain and the future just like the past would be present before its eyes" (Laplace 1902).

The epistemic overtones, and in particular notions like prediction or calculations, brought in side-issues to the debate. Thus, Montague's model-theoretic analysis of (in)determinism was a considerable achievement, freeing the notion from the epistemological load. His analysis, however, is carried out in terms of a set of models of a given theory, and thus forbids asking if a particular model of the theory is deterministic or not. It is exactly this question that we think is essential for deciding whether or not a theory is deterministic. In a similar vein, the analysis does not yield any insight concerning where indeterminism occurs. ${ }^{34}$ Importantly, the Montague notion of indeterminism (as well is its close cousins, Lewis's and Earman's notions) is non-modal in itself. Saying "in itself" we mean that it is not ruled out that a set of models of a given theory can be used to construct a structure that could serve as a semantic theory for a language with modalities. (For a discussion of modal vs. non-modal concepts of (in)determinism, cf. Müller (2009).)

The good news, however, is that things changed considerably for the better! There is no need to shun open possibilities, or to de-epistemologize Laplace, since there are now rigorous theories such as BT of Prior and Thomason, or Belnap's BST that permit cogent theorizing about an open future of possibilities. Moreover, we claim that particular models of BST, namely, MBS's, that we developed here do justice in a thoroughly intuitive way to the most usual intuition of indeterminism. ${ }^{35}$ We specifically mean to include the intuitions of the most hard-headed of scientists when they think about experiment and intervention. Thus, we tend to say, if no physical theory exhibits the kind of usual indeterminism that branching attempts (we think successfully) to capture, so much the worse for indeterminism of physical theories!

Can one relate indeterminism in the sense of Montague-Lewis-Earman (M-L-E) to the usual indeterminism, that is, to a modal concept phrased in terms of open possibilities? The challenge for Earman is to construct, out of a set of models of a given theory that witnesses its indeterminism (in M-L-E sense), a structure that could serve as a semantic theory for a language with modalities. There are indications, such as

\footnotetext{
34 This reminds one of Lewis's desire for a worked-out theory of localized indeterminism as a background for his theory of counterfactuals. Having seen none, he turned to Montague's analysis, or so his letter to Bennett (quoted by Bennett (2003, p. 204)) suggests.

35 The only non-intuitive and purely technical axiom is the Chain Condition 7, which is not needed in finite cases.
} 
Müller's (2007) BT model for quantum mechanics (in the consistent history formulation) that the task is doable, though hard. It is necessary to carry out this task, however, if one wishes to show that various cases of indeterminism (in the sense of $\mathrm{M}-\mathrm{L}-\mathrm{E}$ ) of physical theories, have anything to do with the intuitive notion of indeterminism. Of course, the other option for Earman is to forswear that his ensemble analysis of indeterminism has anything to do with the intuitive (modal) notion.

\section{Final}

$\mathrm{BST} / \mathrm{BT}$ are attempts to do metaphysics in a mathematically rigorous way, with the desideratum: be compatible with current physical theories. There have been some successes: MBS's have histories isomorphic to Minkowski spacetime. There are BST models representing EPR correlations, the Bell-Aspect experiment, or the GHZ and GHSZ theorems. BT offers a reading of the consistent histories theory of quantum mechanics. An open task looms large: construct BST-like models that take into account the fundamental ideas of GR.

Acknowledgments We are indebted to Bryan Roberts and Balázs Gyenis for timely readings of our manuscript and for their comments and corrections. We would also like to thank one referee of this journal for their substantive and detailed comments. TP gratefully acknowledges the support of the Jagiellonian University WRBW2010 grant and of the MNiSW research grant K/PMN/000034.

Open Access This article is distributed under the terms of the Creative Commons Attribution Noncommercial License which permits any noncommercial use, distribution, and reproduction in any medium, provided the original author(s) and source are credited.

\section{References}

Belnap, N. (1992). Branching space-time. Synthese 92, 385-434. See also Belnap (2003a).

Belnap, N. (2002a). Double time references: Speech-act reports as modalities in an indeterminist setting. In F. Wolter, H. Wansing, M. de Rijke, \& M. Zakharyaschev (Eds.), Advances in modal logic (Vol. 3, pp. 37-58). Singapore: World Scientific.

Belnap, N. (2002b). EPR-like "funny business" in the theory of branching space-times. In T. Placek \& J. Butterfield (Eds.), Nonlocality and modality. NATO Science Series (pp. 293-315). Dordrecht: Kluwer.

Belnap, N. (2003a). Branching space-time, postprint, January, 2003. http://philsci-archive.pitt.edu/archive/ $00001003 /$.

Belnap, N. (2003b). No-common-cause EPR-like funny business in branching space-times. Philosophical Studies, 114, 199-221.

Belnap, N. (2005a). Agents and agency in branching space-times. In D. Vanderveken (Ed.), Logic, Thought, and Action (pp. 291-313). Dordrecht: Springer.

Belnap, N. (2005b). A theory of causation: Causae causantes (originating causes) as inus conditions in branching space-times. British Journal for the Philosophy of Science, 56, 221-253. A preprint of this essay may be obtained from http://philsci-archive.pitt.edu/archive/00000891/.

Belnap, N. (2007). An indeterminist view on the parameters of truth. In T. Müller (Ed.), Philosophie der Zeit (pp. 87-113). Klostermann: Frankfurt a.M.

Belnap, N., \& Green, M. (1994). Indeterminism and the thin red line. In J. Tomberlin (Ed.), Philosophical perspectives (Vol. 8, pp. 365-388). Atascadero, CA: Ridgeview Publishing Company.

Belnap, N., Perloff, M., \& Xu, M. (2001). Facing the future: Agents and choices in our indeterminist world. Oxford: Oxford University Press.

Bennett, J. (2003). A philosophical guide to conditionals. Oxford: Oxford University Press. 
Earman, J. (1986). A primer on determinism. Dordrecht: D. Reidel Publishing Company.

Earman, J. (2007). Aspects of determinism in modern physics. In J. Butterfield \& J. Earman (Eds.), The philosophy of physics, Vol. 2 of the Handbook of the philosophy of science. Amsterdam: Elsevier/North-Holland.

Earman, J. (2008). Pruning some branches from branching spacetimes. In D. Dieks (Ed.), The ontology of spacetime II (Chap. 10, pp. 187-206). Amsterdam: Elsevier.

Haag, R. (1990). Fundamental irreversibility and the concept of events. Communications in Mathematical Physics, 132, 245-251.

Hall, G. S. (2004). Symmetries and curvature structure in general relativity. Singapore: World Scientific Publishing.

Kaplan, D. (1989). Demonstratives: An essay on the semantics, logic, metaphysics, and epistemology of demonstratives and other indexicals; and Afterthoughts. In J. Almog, J. Perry, \& H. Wettstein (Eds.), Themes from Kaplan (pp. 481-563, 565-614). Oxford: Oxford University Press.

Laplace, P. S. (1902). A philosophical essay on probabilities. London: Wiley.

Lewis, D. (1986). On the plurality of worlds. Oxford: Basil Blackwell.

MacFarlane, J. (2003). Future contingents and relative truth. The Philosophy Quarterly, 53, 321-336.

Montague, R. (1962). Deterministic theories. In Decisions, values, and groups 2 (pp. 325-370). Oxford: Oxford University Press. (Reprinted in Formal philosophy: selected papers of Richard Montague, edited and with an introduction by R. H. Thomason, New Haven: Yale University Press, 1974).

Müller, T. (2002). Branching space-time, modal logic and the counterfactual conditional. In T. Placek \& J. Butterfield (Eds.), Nonlocality and Modality. NATO Science Series (pp. 273-291). Dordrecht: Kluwer.

Müller, T. (2005). Probability theory and causation: A branching space-times analysis. British Journal for the Philosophy of Science, 56, 487-520.

Müller, T. (2007). Branch dependence in the 'consistent histories' approach to quantum mechanics. Foundations of Physics, 37(2), 253-276.

Müller, T. (2009). Eliminating modality from the determinism debate? Models vs. equations of physical theories. In A. Hieke \& H. Leitgeb (Eds.), Reduction-Abstraction-Analysis. Proceedings of the 31 th international Ludwig Wittgenstein-symposium in Kirchberg, 2008 (pp. 47-62). Frankfurt: Ontos Verlag.

Müller, T., Belnap, N., \& Kishida, K. (2008). Funny business in branching space-times: Infinite modal correlations. Synthese, 164, 141-159.

Norton, J. D. (2008). The dome: An unexpectedly simple failure of determinism. Philosophy of Science, 75, 786-799.

Øhrstrøm, P. (2009). In defence of the thin red line: A case for ockhamism. Humana.Mente, 8, 17-32.

Øhrstrøm, P., \& Hasle, P. F. V. (1995). Temporal logic: From ancient ideas to artificial intelligence. Dordrecht: Kluwer.

Perloff, M., \& Belnap, N. (2010). Future contingents and the battle tomorrow. Review of Metaphysics, forthcoming.

Placek, T. (2000). Is nature deterministic? A branching perspective on EPR phenomena. Cracow: Jagiellonian University Press.

Placek, T. (2004). Quantum state holism: A case for holistic causation. Studies in History and Philosophy of Modern Physics, 35(4), 671-692.

Placek, T. (2010). Possibilities without possible worlds/histories. Journal of Philosophical Logic. doi:10. 1007/s10992-010-9159-x, forthcoming.

Placek, T., \& Müller, T. (2007). Counterfactuals and historical possibility. Synthese, 154(2), 173-197.

Placek, T., \& Wroński, L. (2009). On infinite EPR-like correlations. Synthese, 167(1), 1-32.

Popper, K. R. (1982). The open universe. London: Routledge.

Prior, A. N. (1967). Past, present, and future. Oxford: Oxford University Press.

Thomason, R. H. (1970). Indeterminist time and truth-value gaps. Theoria, 36, 264-281.

Weiner, M., \& Belnap, N. (2006). How causal probabilities might fit into our objectively indeterministic world. Synthese, 149(1), 1-36.

Wroński, L., \& Placek, T. (2009). On Minkowskian branching structures. Studies in History and Philosophy of Modern Physics, 40, 251-258.

Xu, M. (1997). Causation in branching time (i): Transitions, events and causes. Synthese, 112, 137-192. 